\author{
Banco de México \\ Documentos de Investigación
}

\author{
Banco de México \\ Working Papers
}

$N^{\circ} 2018-10$

\title{
Medición de la Actividad Económica en las Principales Zonas Turísticas de Playa en México a través de la Luminosidad Fotografiada desde el Espacio
}

\author{
Irving Llamosas-Rosas \\ Banco de México
}

\author{
Erick Rangel González \\ Banco de México
}

\section{Maritza Sandoval Bustos \\ Banco de México}

Julio 2018

La serie de Documentos de Investigación del Banco de México divulga resultados preliminares de trabajos de investigación económica realizados en el Banco de México con la finalidad de propiciar el intercambio y debate de ideas. El contenido de los Documentos de Investigación, así como las conclusiones que de ellos se derivan, son responsabilidad exclusiva de los autores y no reflejan necesariamente las del Banco de México.

The Working Papers series of Banco de México disseminates preliminary results of economic research conducted at Banco de México in order to promote the exchange and debate of ideas. The views and conclusions presented in the Working Papers are exclusively the responsibility of the authors and do not necessarily reflect those of Banco de México. 
Documento de Investigación 2018-10

Working Paper

2018-10

\section{Medición de la Actividad Económica en las Principales Zonas Turísticas de Playa en México a través de la Luminosidad Fotografiada desde el Espacio}

\author{
Irving Llamosas-Rosas ${ }^{\dagger}$ \\ Banco de México
}

\author{
Erick Rangel González ${ }^{\ddagger}$ \\ Banco de México
}

\section{Maritza Sandoval Bustos ${ }^{\S}$ \\ Banco de México}

Resumen: Basado en fotografías satelitales de luz nocturna de la NASA y el Departamento de Defensa de los Estados Unidos, y usando la metodología propuesta por Henderson, Storeygard y Weil (2012), el presente documento de investigación mide el crecimiento económico de las principales 15 zonas turísticas de playa en México para el periodo 1993 al 2017. Esta metodología ofrece una nueva serie de oportunidades para la medición económica de coyuntura en nuestro país, al proveer de nuevas herramientas para la medición de los cambios en la actividad económica a nivel regional, independientemente de las fronteras administrativas.

Palabras Clave: PIB, luminosidad, satélites, zonas turísticas

Abstract: Based on satellite photos of night light from NASA and the U.S. Department of Defense, and using the methodology proposed by Henderson, Storeygard and Weil (2012), this paper measures the economic growth of the main 15 beach tourist areas in Mexico for the period from 1993 to 2017. This methodology opens a new series of opportunities to measure economic activity, by providing new tools for measurement at the regional level, regardless of administrative boundaries.

Keywords: GDP, lights, satellites, touristic areas

JEL Classification: E01, E23, O11, O47

† Dirección General de Investigación Económica, Banco de México. Correo electrónico: illamosasr@banxico.org.mx.

‡ Dirección General de Investigación Económica, Banco de México. Correo electrónico: erick.rangel@banxico.org.mx.

$\S$ Dirección General de Investigación Económica, Banco de México. Correo electrónico: msandova@banxico.org.mx. 


\section{Introducción.}

En los últimos años, el sector turístico se ha identificado como un impulsor del crecimiento económico. En 2017, este sector llegó a representar el 8.7 por ciento del total de la actividad económica del país. ${ }^{1}$ Asimismo, presentó de 2014 a 2017 una tasa media anual de crecimiento de 3.13 por ciento, superior a la del PIB total de la economía de 2.72 por ciento durante el mismo periodo.

Según el ranking de la Organización Mundial de Turismo (OMT), México se posicionó entre los 9 países con mayor número de llegadas de turistas extranjeros que pernoctaron a nivel mundial durante 2014, posición que mejoró al ocupar el octavo lugar en el año 2016. Adicionalmente, este sector es una fuente importante de divisas. En efecto, el turismo receptivo generó 21,333 millones de dólares en 2017, lo cual es equivalente al $71.8 \%$ de la inversión extranjera directa y al 74.1\% del flujo de remesas que ingresaron al país en ese año.

El dinamismo de la actividad turística se ha reflejado en mayor medida en los destinos de playa, los cuales contribuyeron con el $73.0 \%$ del crecimiento en la demanda total de servicios de hospedaje durante $2017 .^{2}$ No obstante, su impacto en las economías locales no se limita a este tipo de servicios. En efecto, el crecimiento de la actividad turística genera efectos de derrama hacia otros sectores de la economía tales como el comercio, los servicios de transporte, la construcción, entre otros. A pesar de ello, no existen estimaciones oficiales que permitan analizar el desempeño económico a un nivel menos agregado que el correspondiente a las entidades federativas; en particular, para las zonas turísticas de playa más importantes del país, las cuales no necesariamente coinciden con definiciones geográficas de tipo administrativo o político (por ejemplo, municipios, entidades federativas).

El objetivo del presente documento de investigación es estimar el crecimiento económico de las principales zonas turísticas de playa en el país durante el periodo 1993 - 2017 utilizando la metodología desarrollada por Henderson, Storeygard y Weil (2012), la cual parte del hecho de que existe una correlación directa y positiva entre la actividad económica y la luminosidad (observada desde el espacio) en un área geográfica determinada.

\footnotetext{
${ }^{1}$ En la medición más amplia de la Cuenta Satélite del Turismo, el PIB turístico (PIBT).

${ }^{2}$ Según datos de la Organización Mundial de Turismo (OMT).
} 
Las imágenes satelitales de luminosidad que se utilizan provienen de dos fuentes de información: el Departamento de Defensa de Estados Unidos (DoD, por sus siglas en inglés) para el periodo 1993-2013; y la Administración Nacional de la Aeronáutica y del Espacio (NASA, por sus siglas en inglés) para el periodo 2014 - 2017. Las imágenes empleadas fueron capturadas en cada locación del planeta en algún instante entre las 8:30 p.m. y 10:00 p.m. (hora local).

La unidad de observación de los datos es el pixel que corresponde a un área geográfica equivalente a 0.88 kilómetros cuadrados (en el ecuador del planeta) para el caso del DoD, y 0.44 kilómetros cuadrados para los datos de la NASA. Así una de las principales virtudes de la información y la metodología empleada es que permite obtener estimaciones sobre el desempeño económico de cualquier zona del país para la que no existen mediciones oficiales, incluso con independencia de la división político-administrativa, como es el caso de la zona turística de Puerto Vallarta-Riviera Nayarit, la cual se localiza en dos entidades federativas distintas: Jalisco y Nayarit. Otra de las ventajas que tiene la información empleada es que se encuentra disponible de manera más oportuna y con una periodicidad mensual para los datos satelitales más recientes.

Si bien existen algunos trabajos que han analizado los determinantes del crecimiento turístico en México, o que analizan el impacto demográfico y urbano del crecimiento en la actividad turística en algunas zonas del país, no existen trabajos que realicen mediciones del crecimiento económico en las zonas turísticas de playa más importantes del país.

El documento se encuentra organizado de la siguiente forma: en la siguiente sección se presenta una breve revisión de la literatura. Posteriormente, aparece la descripción de los datos. La metodología está contenida en la sección 4, mientras que los resultados de la investigación se muestran en el apartado 5. Si bien no es objeto del presente trabajo analizar los determinantes del crecimiento económico en las distintas zonas analizadas, en la sección 6 se presenta una discusión de algunos de los factores que podrían explicar la heterogeneidad en los crecimientos registrados en los destinos de playa. Finalmente, se exponen las conclusiones. 


\section{Revisión de la Literatura}

Rassy y Smith (2013) analizaron el impacto económico derivado del brote de influenza $\mathrm{A}(\mathrm{H} 1 \mathrm{~N} 1)$ en los 10 principales destinos de pasajeros en México: Los Cabos, Ciudad de México, Acapulco Zihuatanejo, Guadalajara, Puerto Vallarta, Monterrey, Cancún, Cozumel y Mazatlán. Siendo los destinos de playa ubicados principalmente en Quintana Roo y Baja California sur los más afectados en términos porcentuales con respecto a su PIB.

Por su parte, Cárdenas (2016) estudia el crecimiento urbano y demográfico debido al auge del Turismo en las ciudades de Acapulco, Guerrero; Cancún, Quintana Roo y Puerto Vallarta, Jalisco. Sin embargo, la autora se centra en cifras poblacionales, hectáreas urbanizadas y cuestiones de planeación urbana.

Guzmán et al. (2011) emplean un modelo autoregresivo para el periodo 1980-2009 para identificar los factores que influyen sobre la demanda internacional de turismo en México, sus resultados indican que dicha demanda responde inelásticamente a cambios en el costo de vida (medido a través del INPC) en México, y que el crecimiento económico de Estados Unidos y Canadá influyen positivamente sobre el turismo receptivo. Asimismo, encuentran un efecto positivo de la promoción que realice el turista que visitó el periodo anterior sobre la variable de análisis. Resultados similares son encontrados por Hernández (2011), cuyas estimaciones indican que el PIB de los Estados Unidos y los valores rezagados de arribo de turistas internaciones influyen positivamente sobre el turismo receptivo.

Otro estudio similar es el de Sanchez y Cruz (2016), quienes emplean un modelo estructural de vectores autoregresivos para el periodo 1995-2015 y encuentran un efecto positivo y estadísticamente significativo del crecimiento económico de los Estados Unidos y de las variaciones del tipo de cambio peso-dólar americano sobre la llegada de visitantes internacionales a México.

Montaño et al (2012) analizan el efecto de las crisis económicas internacionales sobre diversos indicadores del turismo a nivel nacional para el periodo 1990-2010. Asimismo, estudian la correlación del crecimiento del turismo internacional con el ciclo de vida de los tres destinos turísticos de playa más importantes en México (Los Cabos, Cancún y Puerto Vallarta). 
Como puede apreciarse, no existen estudios previos que estimen el crecimiento económico de los principales destinos turísticos de playa en México, ni empleando la misma metodología aplicada en el presente documento o alguna otra. Sin embargo, sí hay varios estudios internacionales que aplican la luminosidad capturada por fotografías satelitales para estimar el desempeño económico en diversas zonas o regiones. Por ejemplo, Henderson Soterygard y Weil (2012), artículo empleado como base en este estudio, utilizan la metodología para estimar el Producto Interno Bruto (PIB) de países que no cuentan con "buena calidad" en sus datos, para comparar el crecimiento económico en las zonas costeras contra el crecimiento en las zonas interiores de África Sub-Sahariana, así como para medir el efecto de la malaria sobre la actividad económica en la citada área africana. En un estudio similar, Chen y Nordhaus (2011) utilizan los datos satelitales del DoD para estimar el Producto Interno Bruto de distintos países del planeta y concluyen que dicha información es útil para realizar estimaciones en países en donde se cuenta con censos económicos e información estadística confiable. La información también ha sido empleada, entre otras cosas, para realizar mapas de la actividad económica regional (Doll et al 2006) y para analizar la geografía urbana de la ciudades (Harari, 2016).

\section{Datos.}

Como se mencionó, la base de datos utilizada de luminosidad satelital provienen de dos fuentes de información: i) el Departamento de Defensa de Estados Unidos (DoD) para el periodo 1993-2013; y ii) la Administración Nacional de la Aeronáutica y del Espacio (NASA) para el periodo 2014 - 2017. Las imágenes empleadas fueron capturadas en cada ubicación del planeta en algún instante entre las 8:30 p.m. y 10:00 p.m. (hora local). Posteriormente, científicos del "National Oceanic and Atmospheric Administration" (NOAA) y del "National Geographic Data Center" procesan los datos, los cuales se hacen disponibles al público en general. ${ }^{3}$

\footnotetext{
${ }^{3}$ El procesamiento de datos incluye correcciones por el ciclo lunar, periodos del año en donde oscurece más tarde, incendios forestales, y auroras boreales, entre otros. Lo anterior con la finalidad de dejar únicamente las luces generadas por el ser humano en las imágenes satelitales. Los datos se encuentran disponibles con una periodicidad anual para la primera fuente mencionada, y con una mensual para la segunda.
} 
Los archivos disponibles para los satélites del Departamento de Defensa de Estados Unidos contienen la información a nivel global, con un tamaño de 2.7 gigabytes por cada archivo/año de información. Para los archivos disponibles por la NASA-NOAA, los datos están disponibles por sección del planeta (la correspondiente a México es la región 1, Norteamérica), y cada uno de ellos (un mes de información) tiene un tamaño de 2 gigabytes, debido primordialmente a la mayor precisión de los satélites utilizados (aproximadamente 4 veces más datos por pixel).

La unidad de observación de los datos es el pixel que corresponde a un área geográfica equivalente a 0.88 kilómetros cuadrados (en el ecuador del planeta) para el caso de la DoD (30 arco-segundos), y 0.44 kilómetros cuadrados para los datos de la NASA (15 arcosegundos). Dentro de cada pixel la luminosidad es medida en una escala de 0 (ausencia total de luz) a 63 (grado máximo de luminosidad) en el caso del DoD, mientras que las imágenes generadas por la NASA no tienen un límite preestablecido. Posteriormente, los datos son reportados de acuerdo a un sistema de coordenadas geográficas angulares (latitud y longitud) y emparejados con la cartografía del INEGI a través del software GIS. La Figura 1 resume el procesamiento de los datos.

\section{Figura 1}

\section{Descripción Gráfica del Procesamiento de Datos}

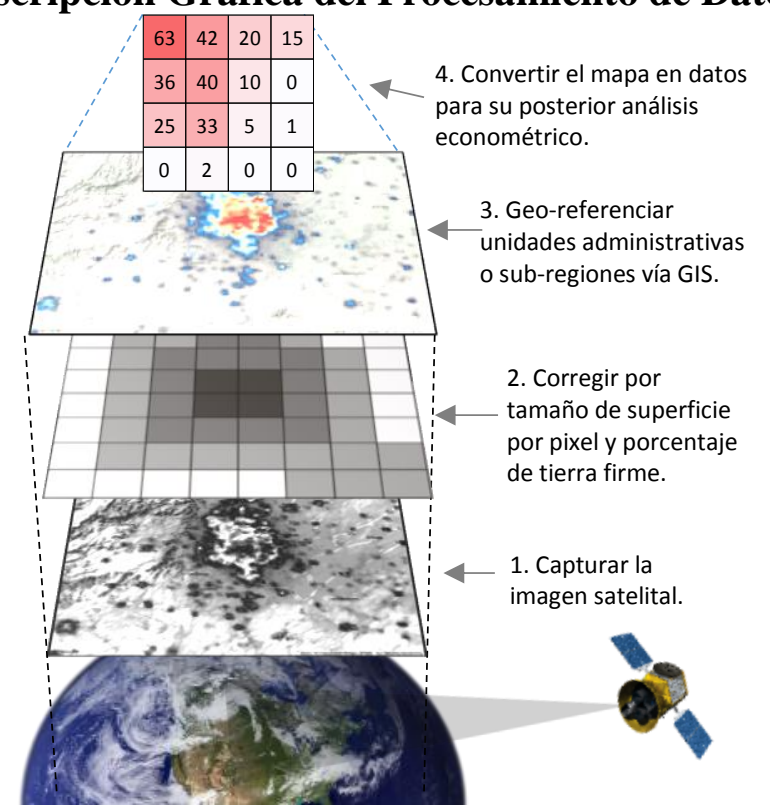

Fuente: Elaboración propia con base en Henderson, Storeygard y Weil (2012). 
Los datos satelitales fueron procesados siguiendo la metodología propuesta por Henderson, Storeygard y Weil (2012) para ajustar el cambio en el tamaño del pixel debido a la curvatura del Planeta, así como tomar en consideración los reflejos de las luces en campos de agua y litorales.

Cabe destacar que el ajuste toma en consideración los ríos y lagos, al eliminar los pixeles que se encuentran enteramente dentro de estos espacios aunque registren presencia de luz. Asimismo, se hace una corrección a los casos donde el pixel se encuentre en los bordes del litoral (por ejemplo, que parte del pixel se encuentre en tierra firme y la otra parte en un lago, mar o río) multiplicando la luz registrada en dicho pixel por el área en kilómetros cuadrados de tierra firme registrado en el Gridded Population of the World versión 3 (GPWv3) el cual registra las áreas de tierra firme en kilómetros cuadrados para cada pixel a una resolución de 30 arcosegundos.

El ajuste final se realiza incorporando los mapas de las unidades administrativas (entidad, municipio, cualquier sub-región ad-hoc) al ajuste de luz y kilómetros cuadrados de tierra firme, sumando todos los pixeles dentro de una misma unidad. Para recuperar los niveles de intensidad por kilómetro cuadrado, se divide la suma por el área total de la unidad.

Para poder realizar el análisis con respecto a los niveles de actividad económica, se relacionan los niveles de luminosidad por entidad federativa con la metodología anteriormente expuesta con los niveles del PIB para cada entidad federativa de 1992 al 2013. Dicha relación es loglinear y se muestra en la Gráfica 1.

En la gráfica resaltan los casos de la Ciudad de México y Campeche, por lo que resulta indispensable incorporar efectos fijos dentro del análisis. Para el caso de Campeche este resultado se deriva de la influencia de la industria petrolera en territorio marítimo, el cual impulsa el PIB por encima de la luminosidad captada en tierra firme. Para el caso de la Ciudad de México, la saturación de luz al máximo de 63 que captura el satélite del Departamento de Defensa evita que se capture la totalidad del PIB que estaría asociada a dicha entidad federativa. 


\section{Gráfica 1 \\ Logaritmo Natural del PIB a Precios Constantes (2008) y Logaritmo Natural de Luz por Entidad Federativa, 1992-2013.}

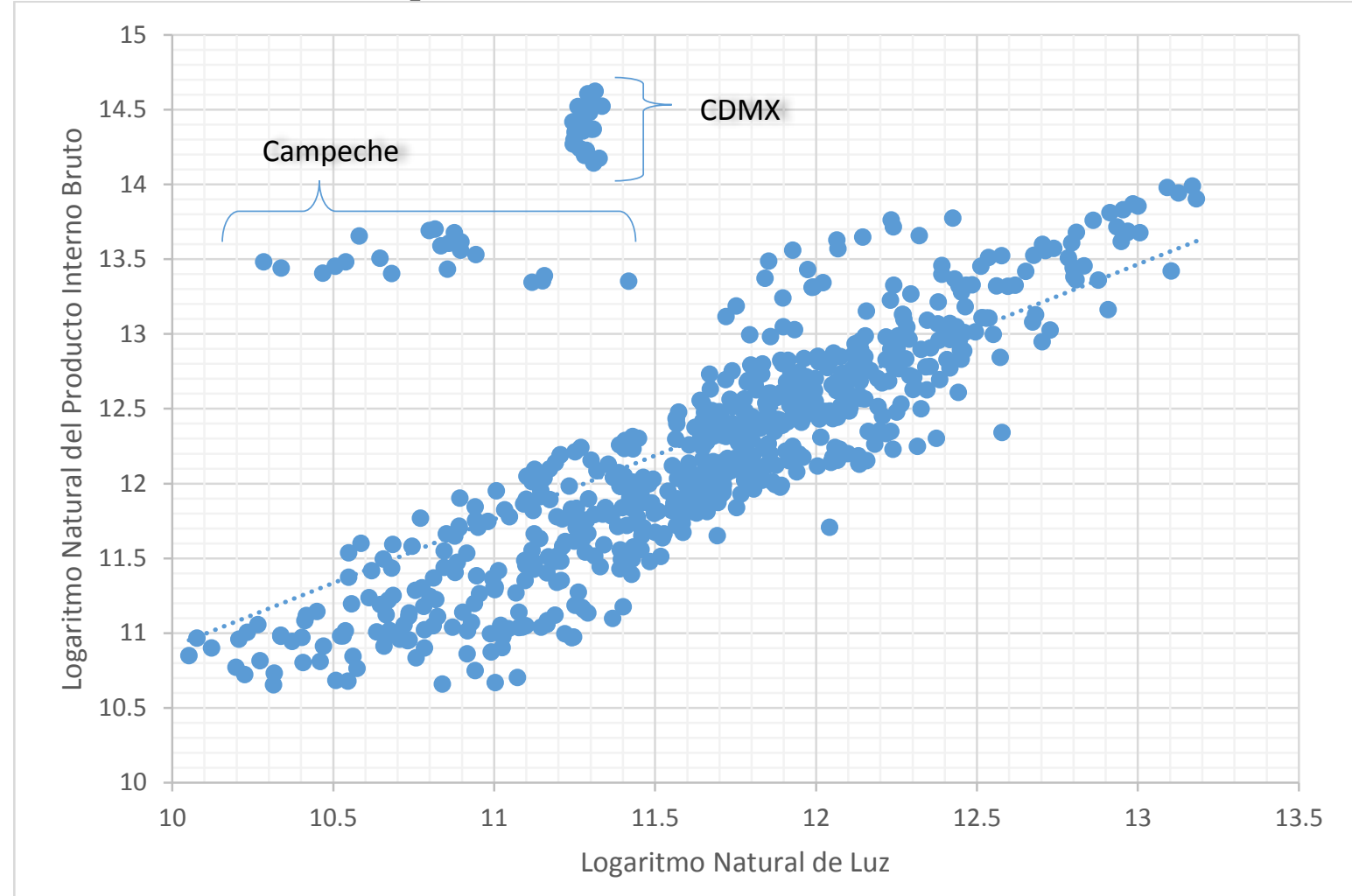

Fuente: Estimaciones propias con base en información del Departamento de Defensa de Estados Unidos.

A manera de ejemplo, la Figura 2 presenta las imágenes de luminosidad del área turística conformada por Cancún y la Riviera Maya para los años 1993 y $2013 .{ }^{4}$ El incremento de la intensidad de la luz asociada a la actividad humana es evidente entre esos años, desarrollándose en toda la zona costera a lo largo de la Riviera Maya.

${ }^{4}$ Dado que las imágenes son cuatro veces más precisas en los satélites de la NASA no es posible comparar visualmente el año inicial con las imágenes de 2017. 


\section{Figura 2}

\section{Imágenes Satelitales de Luminosidad}

Cancún-Riviera Maya, 1993 - 2013

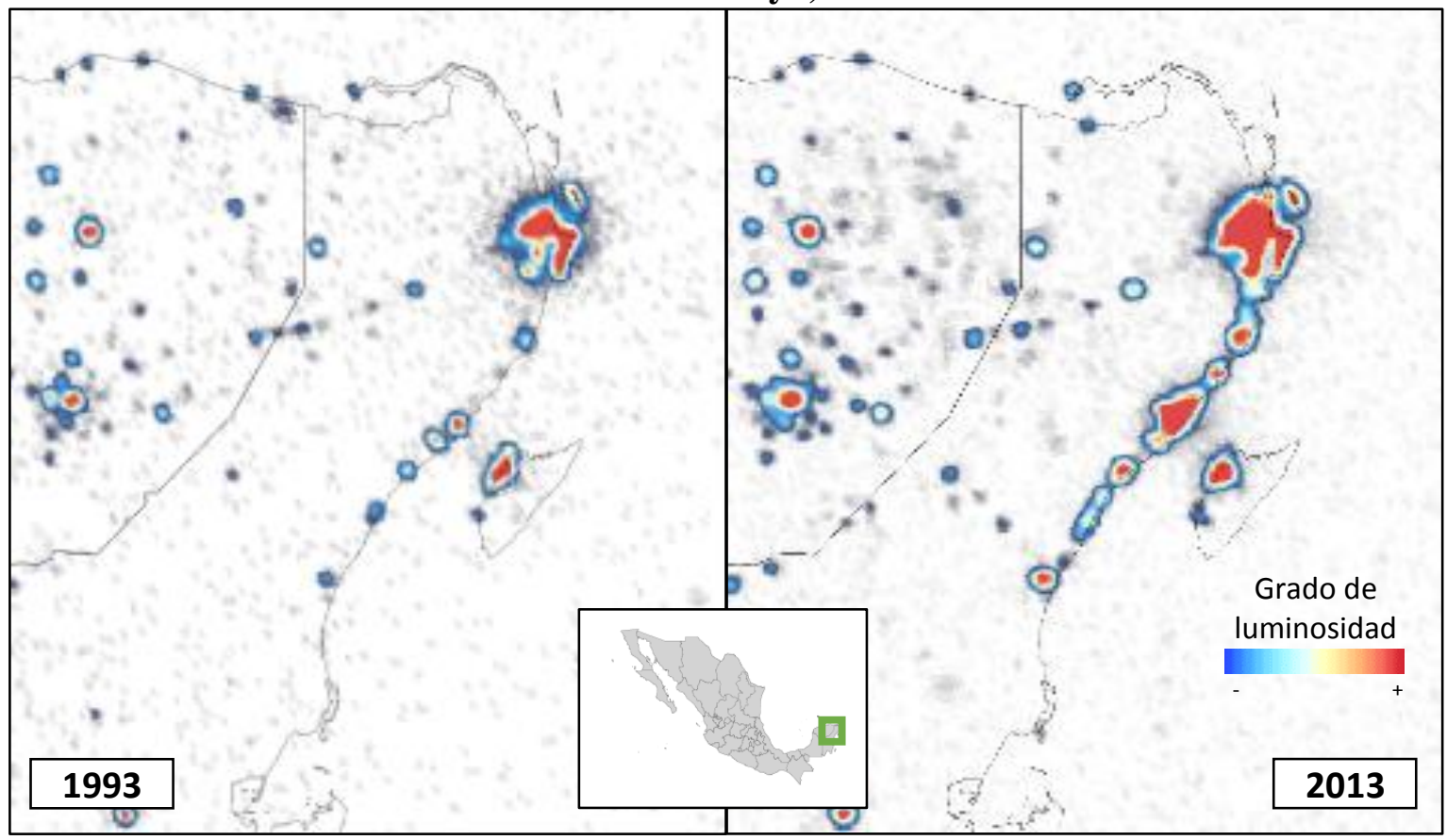

Fuente: Estimaciones propias con base en información del Departamento de Defensa de Estados Unidos.

Así, dado que la información de luminosidad está disponible para cada pixel, es posible obtener estimaciones sobre el desempeño económico de cualquier zona del país, con independencia de la división político-administrativa, como es el caso de la zona turística de Puerto Vallarta-Riviera Nayarit, la cual se localiza en dos entidades federativas: Jalisco y Nayarit (Figura 3). Al respecto, se puede destacar el incremento de luminosidad a lo largo de la costa de Nayarit entre 1993 y 2013, lo cual refleja el desarrollo de Nuevo Vallarta y Punta Mita, así como de la zona de influencia de esos destinos turísticos. 
Figura 3

Imágenes Satelitales de Luminosidad

Vallarta-Riviera Nayarit, 1993 - 2013

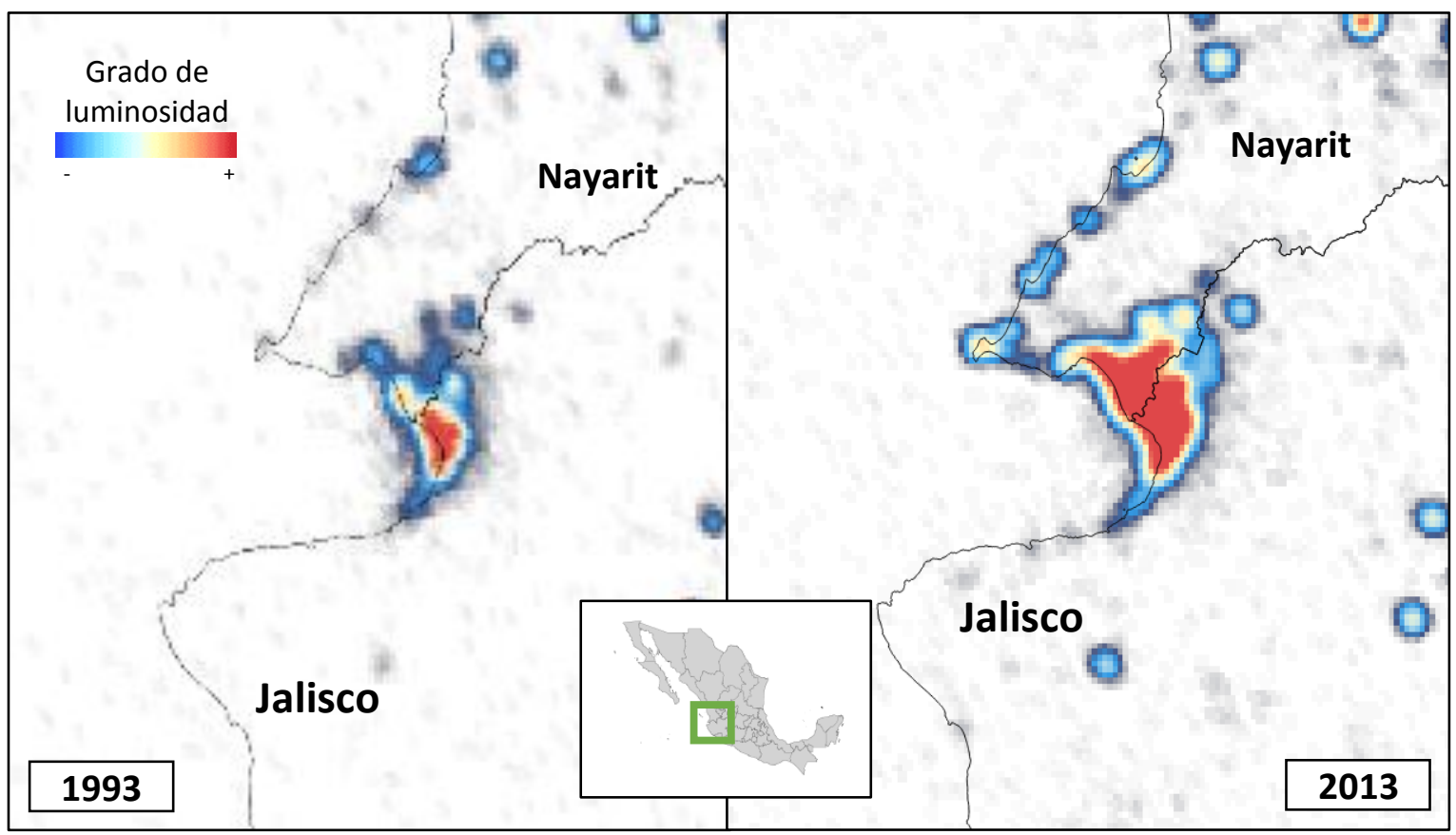

Fuente: Estimaciones propias con base en información del Departamento de Defensa de Estados Unidos.

En la Figura 4 se puede apreciar el incremento substancial que ha tenido la zona turística de Los Cabos, y en la parte superior del mapa se puede apreciar La Paz, destacando el desarrollo de poblaciones sobre la carretera, así como el grado de luminosidad de ambos desarrollos entre los años 1993 y 2013. 
Figura 4

Imágenes Satelitales de Luminosidad

Los Cabos - La Paz, 1993 - 2013

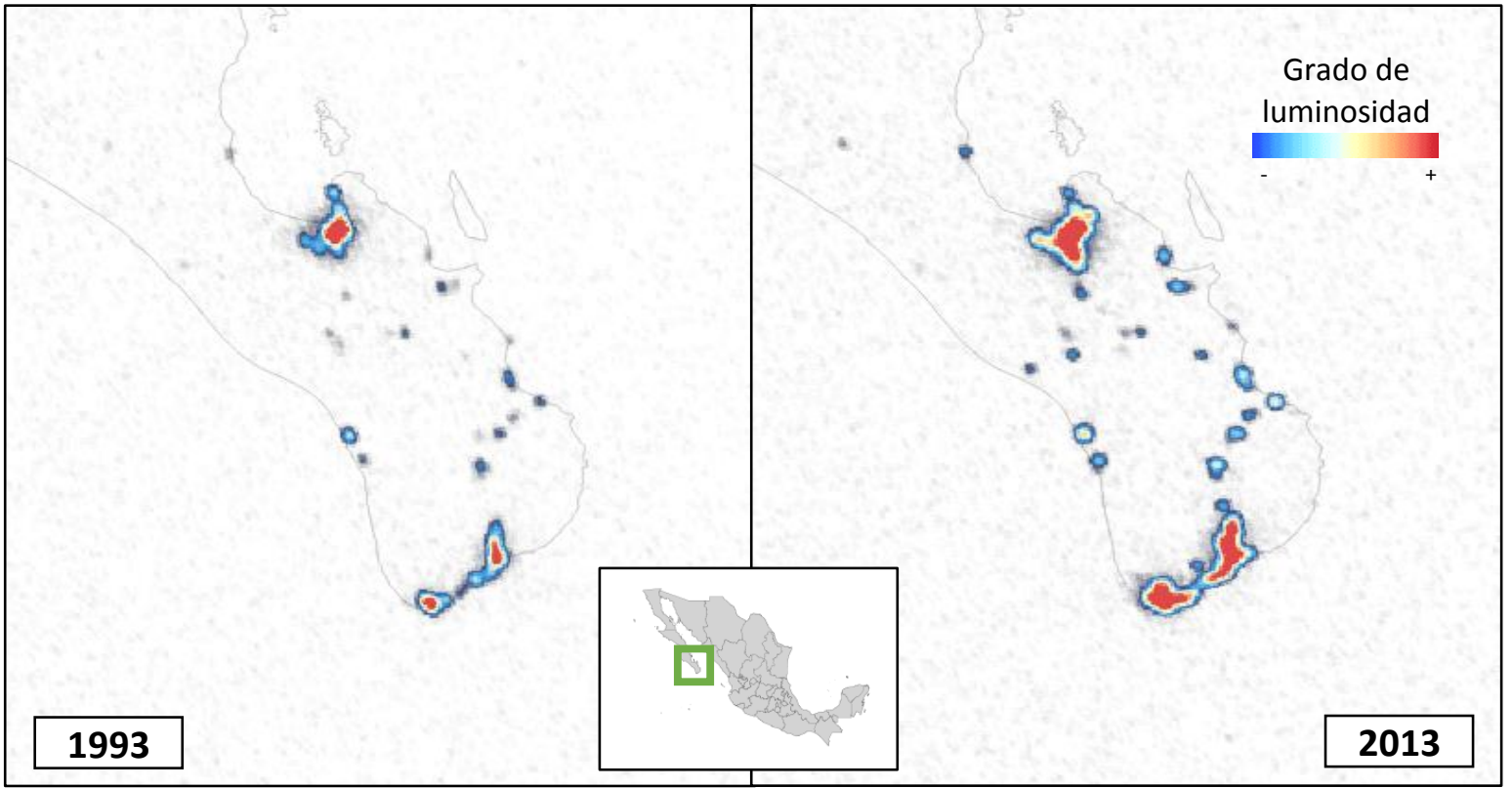

Fuente: Estimaciones propias con base en información del Departamento de Defensa de Estados Unidos.

Aunado a lo anterior, también es posible medir efectos de política pública e infraestructura en la actividad económica por medio de la intensidad luminosa. Por ejemplo, en la segunda mitad del 2013 se inauguró la autopista Durango-Mazatlán, lo que redujo el tiempo de traslado entre ambas ciudades en tres horas e impulsó la reactivación económica de la región, en particular de la zona turística de Mazatlán. La Figura 5 muestra los cambios en luminosidad promedio entre enero y julio de 2013 (previo a la inauguración) y el mismo periodo en 2014. Las variaciones positivas en la luminosidad se registran en color verde, mientras que las negativas se representan en color amarillo. En dicha figura se puede observar un aumento significativo en la intensidad luminosa tanto del puerto de Mazatlán como a lo largo del trayecto de la nueva autopista. Así, es posible inferir que una mejora en la infraestructura carretera se reflejó, a su vez, en un aumento en la luminosidad del destino turístico de playa y, por lo tanto, en su actividad económica. 


\section{Figura 5}

Cambios en la Luminosidad Promedio de la Zona Turística de Mazatlán

Enero - Julio de 2014 - Enero - Julio de 2013

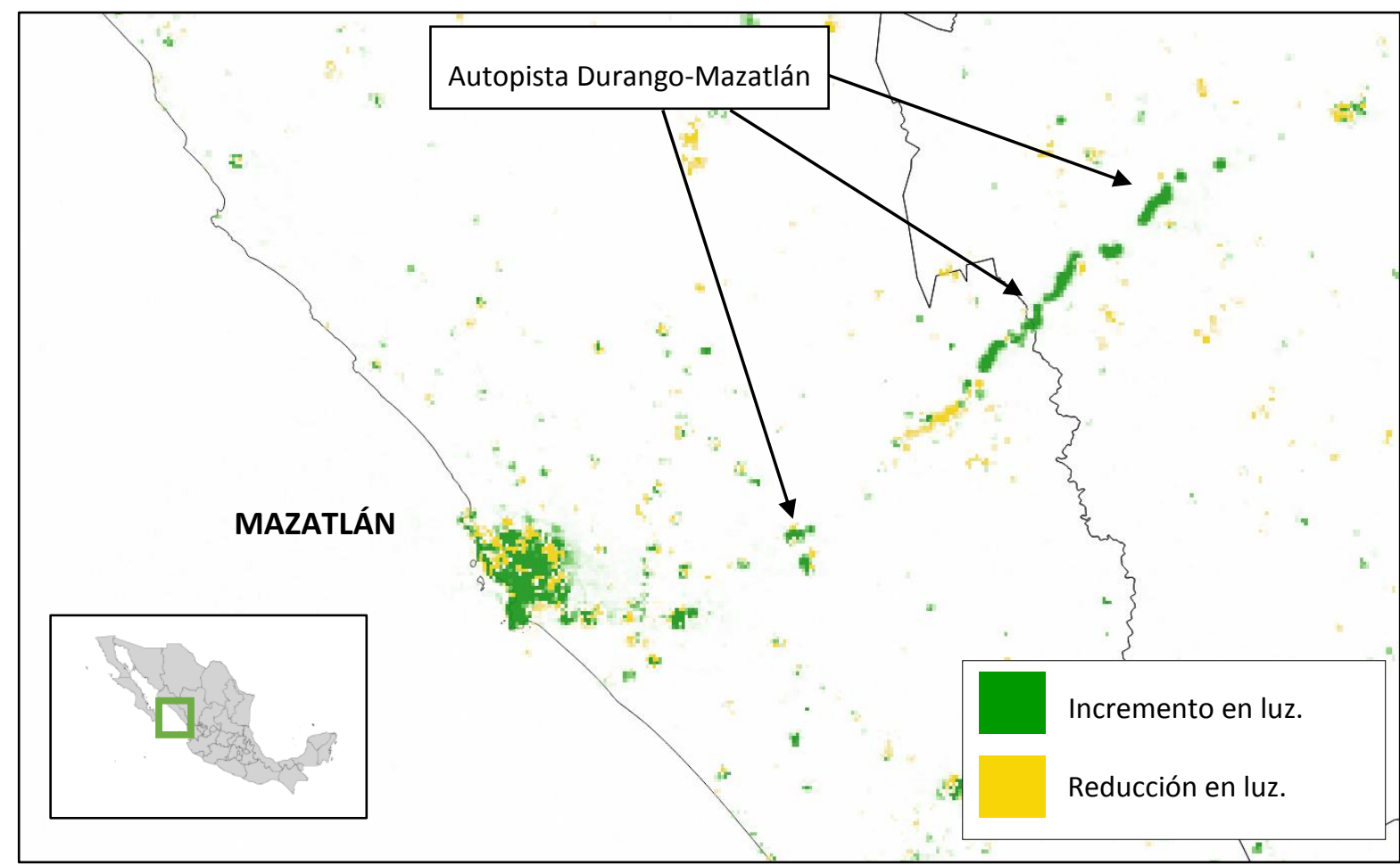

Fuente: Estimaciones propias con base en información del Earth Observation Group, NOAA National Geophysical Data Center.

Para las zonas de playa a estudiar, se seleccionaron los principales 15 centros de playa reportados por el Sistema de Monitoreo DataTur. El reporte proviene de la Secretaría de Turismo y se realiza a 70 centros turísticos a nivel nacional. En la figura 6 se muestra la ubicación de cada una de estas 15 zonas de playa analizadas en el presente documento. 


\section{Figura 6}

\section{Ubicación de las Zonas de Playa Analizadas}

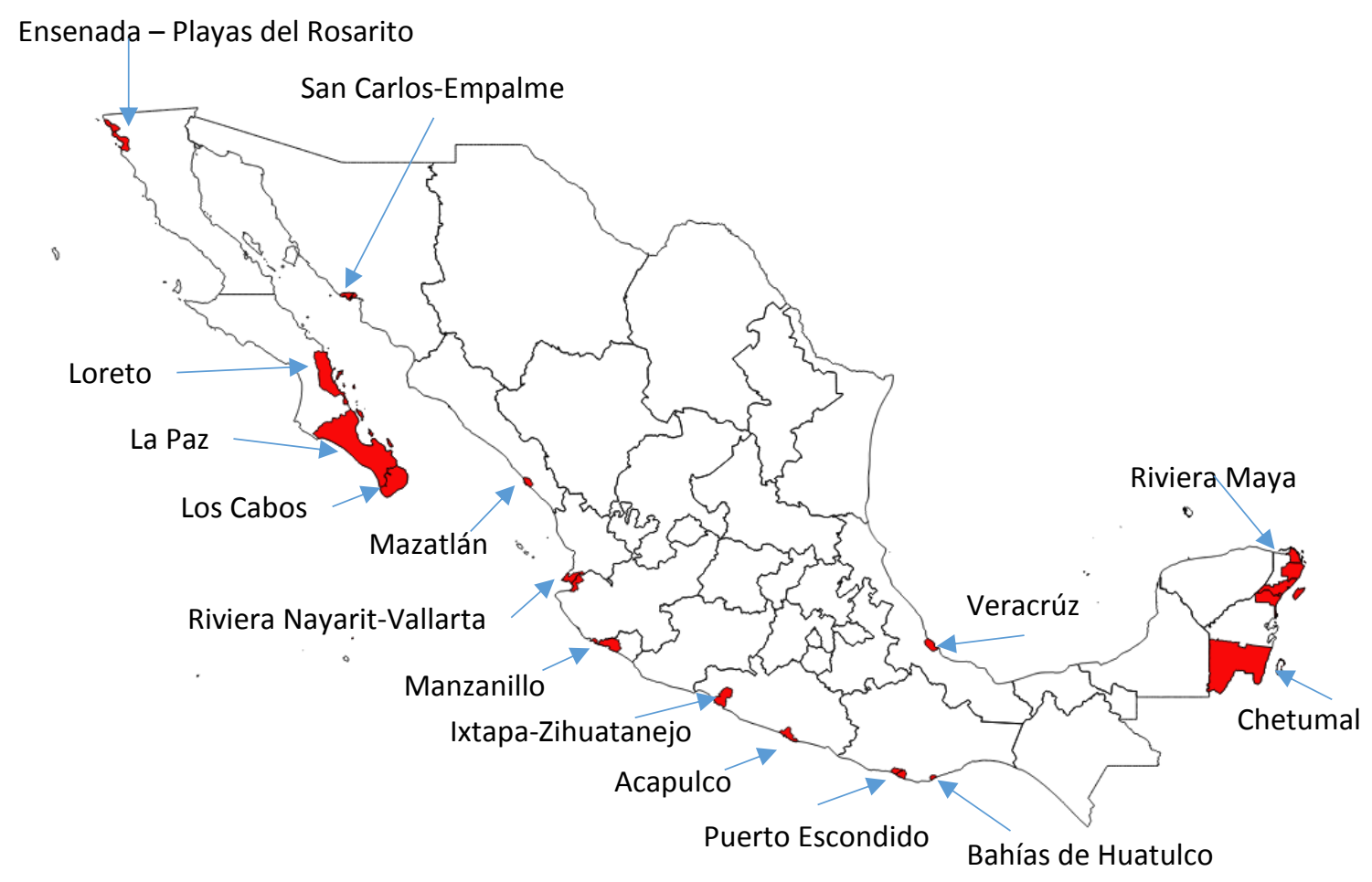

Fuente: Elaboración propia.

Básicamente se toma en consideración la extensión municipal como polígono de estudio, como es en los casos de Cancún - Riviera Maya, el cual se compone de varios municipios. La extensión del municipio no afecta el cálculo, ya que se analiza la suma de intensidades de luz nocturna capturada por satélite, por lo tanto al tener extensiones sin registro de luz, éstas no suman en el cálculo. Cabe aclarar que en otros casos, como el municipio de Acapulco, Guerrero, su extensión geográfica abarca no sólo su núcleo de luz sino también otras poblaciones que generan luminosidad, como la población de Lomas de San Juan, el cual no forma parte propiamente del núcleo de Acapulco aunque si está dentro del polígono municipal. Sin embargo, la luminosidad del centro turístico abarca también parte de los municipios colindantes de Coyuca de Benítez y San Marcos, por lo que se hace prioritario 
ajustar el polígono de análisis para incluir sólo lo correspondiente a la zona de playa. (Ver Figura 7).

Figura 7

Reducción del Polígono de Estudio.

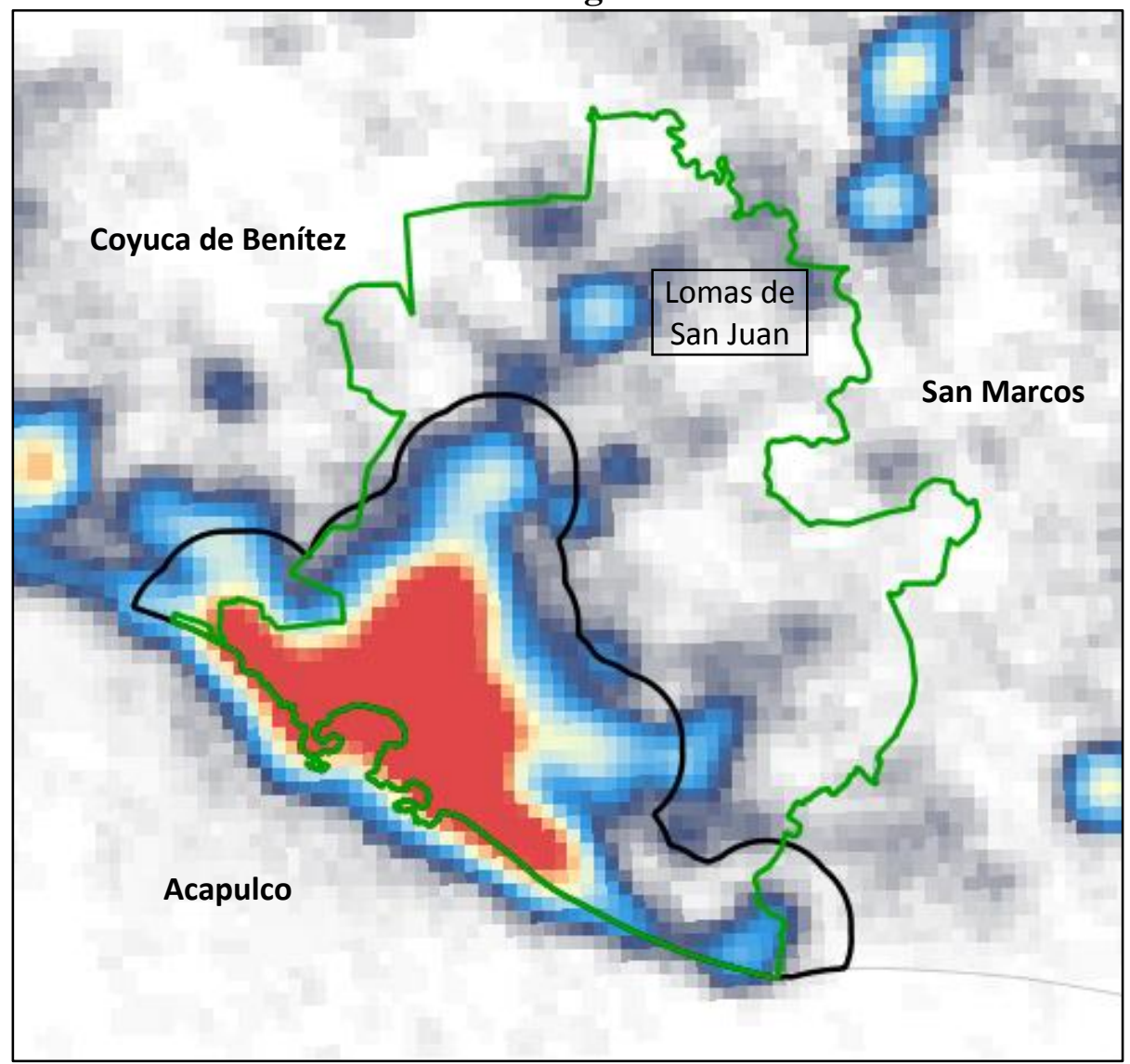

Fronteras en Verde: Polígono Municipal. Frontera en Negro: Polígono reducido.

Fuente: Estimaciones propias con base en información del Earth Observation Group, NOAA National Geophysical Data Center y Extensiones Municipales del INEGI.

En estos casos, se determinó una metodología para reducir el polígono de estudio al núcleo turístico:

a) Se filtran los pixeles con una intensidad luminosa menor a 20 en la imagen satelital más reciente.

b) Del polígono principal de luz, se construye alrededor un área circundante de 5 kilómetros a partir de la última fotografía satelital disponible del DoD. Así, se limita el crecimiento (o decremento) de zonas que no corresponden propiamente al núcleo turístico. 


\section{Figura 8}

Distribución de la luminosidad en los principales destinos de playa, 1993,2003 y 2013

Ixtapa Zihuatanejo

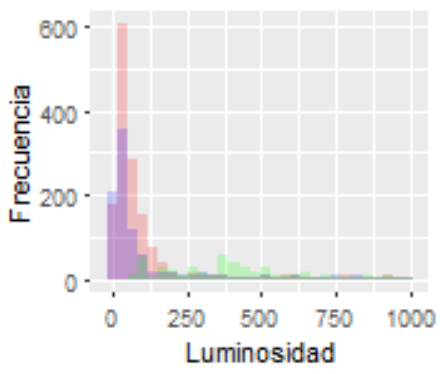

La Paz

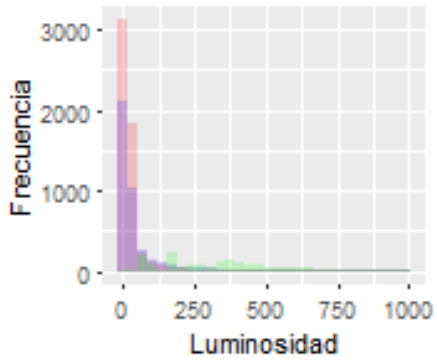

Puerto Escondido

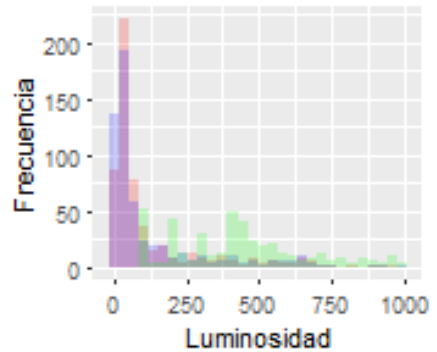

Cancún - Riviera Maya

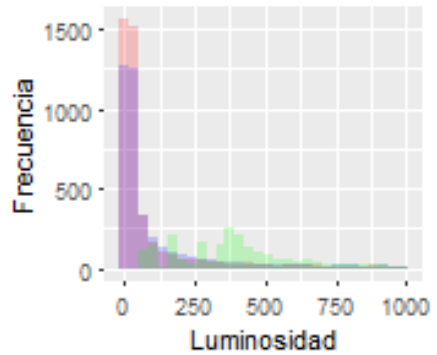

Veracruz-Boca del Río

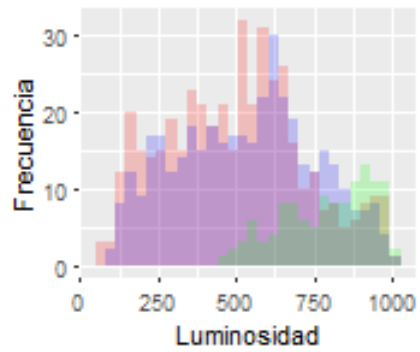

Playas del Rosarito

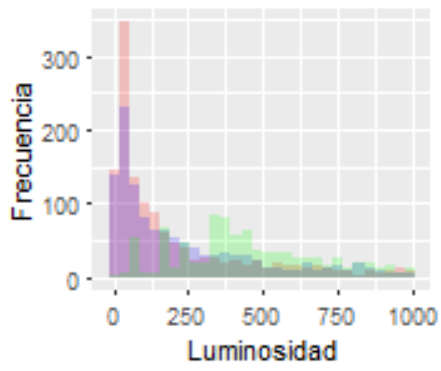

Los Cabos

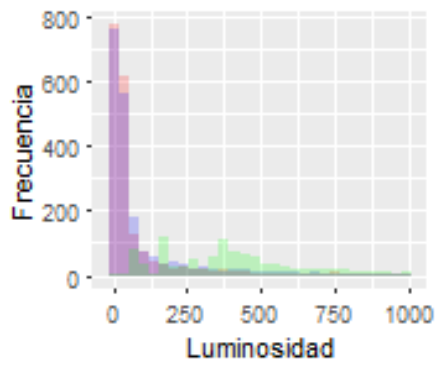

Bahías de Huatulco

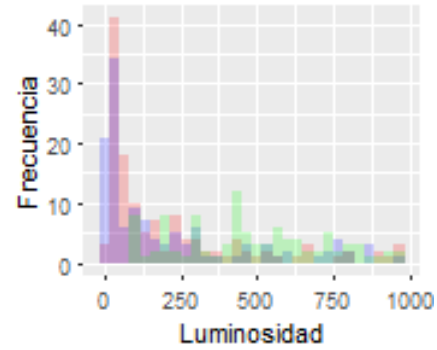

San Carlos-Empalme

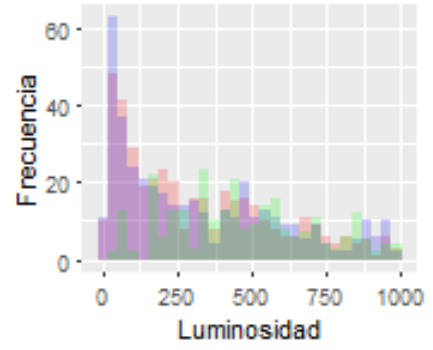

Acapulco

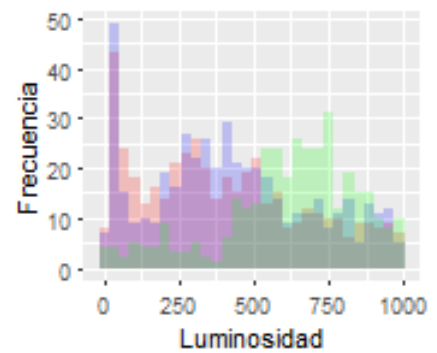

Loreto

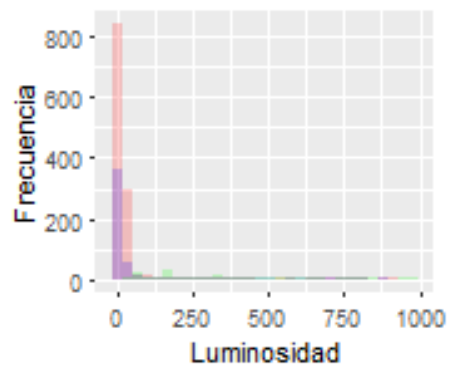

Manzanillo

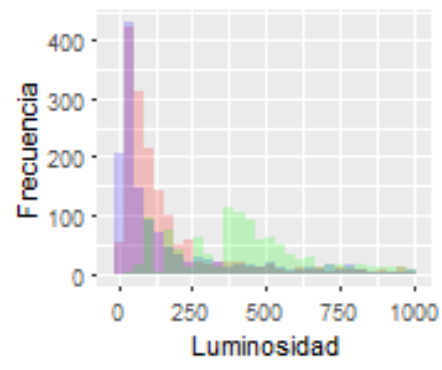

Chetumal

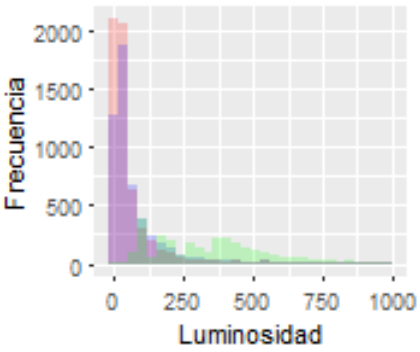

Riviera Vallarta-Nayarit

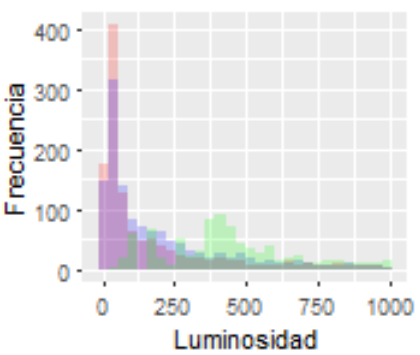

Mazatlán

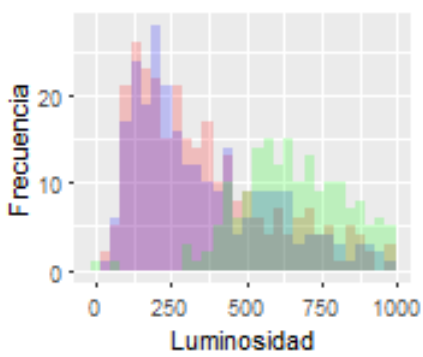

Densidad de Luminosidad x Área del Polígono. Rojo: 1993; Azul: 2003; Verde: 2013.

Fuente: Estimaciones propias con base en información del Earth Observation Group, NOAA National Geophysical Data Center y Extensiones Municipales del INEGI. 
Una vez delimitados los polígonos de estudio, se extrae la información pertinente a cada uno de ellos. En la Figura 8, se muestran las densidades de luz para cada una de las 15 zonas turísticas de playa una vez multiplicadas por el área de tierra firme para tres años, 1993, 2003 y 2013.

Los histogramas mostrados señalan la distribución por grado de luminosidad/área de cada zona turística, en rojo se muestran los datos satelitales en 1993, en azul los datos de 2003 y en verde los de 2013 en transparencias para poder apreciar los tres histogramas. A partir de este análisis estadístico, podemos inferir el gran crecimiento que ha obtenido Cancún-Riviera Maya, ya que las distribuciones pasan de estar concentradas primordialmente en la parte izquierda de la escala en 1993 y 2003, a distribuirse completamente a la derecha (histograma verde).

\section{Metodología.}

Para la estimación del crecimiento económico de las principales zonas turísticas de playa en México se utilizó la metodología desarrollada por Henderson, Storeygard y Weil (2012), la cual parte del hecho de que existe una correlación directa y positiva entre la actividad económica y la luminosidad en un área geográfica determinada. Dicha correlación se explota para estimar el crecimiento económico regional a través del incremento en la luz observada desde el espacio a través del tiempo.

Esta relación se basa en la definición del crecimiento de la actividad económica real (no observable) $y$, el crecimiento medido del PIB a precios constantes medido por las cuentas nacionales como $z$, y el crecimiento de las luces capturadas por los satélites como $x$, donde el subíndice j representa las entidades federativas. Por lo tanto, asumiendo que la medición de la actividad real de la economía se realiza de manera insesgada con un término de error con media cero y varianza $\sigma_{Z}^{2}$ :

$$
z_{j t}=y_{j t}+\varepsilon_{z, j t}
$$

Y también, la correlación entre la actividad económica real y su impacto en el uso de luces está denotada por la siguiente ecuación:

$$
x_{j t}=\beta y_{j t}+\varepsilon_{x, j t}
$$


donde $\beta$ captura la elasticidad de las luces con respecto a la actividad económica real (no observada), en otras palabras, captura el cambio porcentual en el uso de la luz ante un cambio en la actividad económica real. ${ }^{5}$

Debido a que los errores en la medición de la actividad económica real así como los errores en la relación entre dicha actividad y las luces nocturnas, se consideran eventos independientes, por lo que la covarianza entre $\varepsilon_{x}$ y $\varepsilon_{z}$ es igual a cero. Así, es posible combinar (1) y (2) para tener como resultado:

$$
z_{j t}=\psi x_{j t}+e_{j t}
$$

donde $\psi$ es el inverso de la elasticidad entre la actividad económica y las luces nocturnas. Para capturar cambios en los satélites y cambios tecnológicos independientemente del lugar donde se realice la actividad económica, se incluyen variables dicotómicas anuales. Así mismo, para incorporar la diferente mezcla de actividades económicas de cada unidad geográfica (las actividades agropecuarias pueden tener un uso bajo en comparación con manufacturas o el sector servicios), se incorporarán efectos fijos por zona geográfica.

$$
z_{j t}=\psi x_{j t}+c_{j}+d_{t}+e_{j t}
$$

En efecto, al controlar por los efectos anuales, los cuales son comunes a todas las unidades geográficas, así como los efectos fijos, los cuales toman en consideración diferentes mezclas productivas; podremos estimar la relación entre la actividad económica medida por $z_{j t}$ y la luminosidad, medida por $x_{j t}$, la cual es independiente de la región en medición. Por lo tanto, si uno está interesado en calcular el incremento de la actividad económica en cualquier subregión, en donde no se dispone de una medición de la actividad económica, es posible utilizar el coeficiente $\hat{\psi}$ :

$$
\Delta \hat{z}_{k t}=\hat{\psi} \Delta m_{k t}+d_{t-(t-1)}
$$

Donde $\Delta m_{k j}$ es el incremento de la luminosidad en cualquier subregión arbitraria k, la cual, al multiplicarse por el valor estimado $\hat{\psi}$, nos da el incremento del producto en dicha región.

5 Se reconoce que la relación entre uso de luz y actividad económica puede mantener doble causalidad; sin embargo, siguiendo a Henderson et al. (2012) y el supuesto de independencia entre actividad económica y el uso de luces nocturnas, es suficiente utilizar la correlación simple para derivar la expresión (3) sobre el crecimiento del PIB. 
En el presente trabajo, se utilizará esta ecuación para estimar el crecimiento de las principales zonas turísticas de playa de México, con datos del PIB estatal obtenidos del Instituto Nacional de Estadística, Geografía e Informática (INEGI) para los periodos 1992-2015.

\section{Resultados}

La ecuación (4) se estima a través de un modelo lineal con datos en panel y efectos fijos, ${ }^{6} \mathrm{y}$ para fines de comparabilidad también se realizaron las estimaciones a través del método de regresión agrupada (pooled OLS). Cabe señalar que esta última metodología es más restrictiva y puede generar sesgos; sus estimaciones están reportadas en la primer columna del Cuadro 1. Por su parte, los resultados de la estimación de efectos fijos, los cuales incluyen también efectos temporales, están reportados en la segunda columna. Así, es posible inferir que un incremento de $1 \%$ en la luminosidad está asociado con un aumento de $0.227 \%$ en el PIB de las entidades federativas. Este resultado es similar al obtenido por Henderson, Storeygard y Weil (2012) quienes estiman una elasticidad de 0.277 con datos por país a nivel mundial.

\section{Cuadro 1}

Elasticidad del PIB a la Luminosidad, 1993 - 2013

\begin{tabular}{lcc}
\hline Variables: & $(1)$ & $(2)$ \\
\hline $\ln ($ Luz/area) & $0.171^{\star \star \star}$ & $0.227^{\star \star \star}$ \\
& $(0.0283)$ & $(0.0395)$ \\
Constante & $11.907^{\star \star \star}$ & - \\
& $(0.1256)$ & \\
\hline Efectos Fijos por Entidad & & $\checkmark$ \\
Efectos Fijos Temporales & $\checkmark$ & $\checkmark$ \\
Observaciones & 672 & 0.873 \\
$\mathrm{R}^{2}$ & 0.097 &
\end{tabular}

Fuente: Estimaciones propias con base en información del INEGI y el Departamento de Defensa de Estados Unidos.

A partir de los resultados del Cuadro 1, se procedió a calcular el crecimiento económico de las principales zonas turísticas del país de acuerdo a la ecuación 5. Posteriormente, se estimó la tasa media de crecimiento anual del PIB para cada una de las zonas. En específico, se multiplicó la elasticidad obtenida $\widehat{\psi}$ por el crecimiento anual de la luminosidad en los

\footnotetext{
${ }^{6}$ Debido a la heterogeneidad en la estructura productiva de las unidades geográficas (entidades federativas) se asume que Efectos Fijos es más apropiado.
} 
destinos turísticos, y se le agregaron los efectos de tiempo correspondientes, para de ahí estimar la tasa referida durante el periodo $1993-2017 .^{7}$

Una vez estimada la tasa media de crecimiento anual de cada una de las zonas turísticas, esta se normalizó por el promedio simple de dichas tasas, obteniendo así su crecimiento relativo durante el periodo considerado (última columna del Cuadro 2). Un cociente mayor que la unidad indica un crecimiento superior al promedio de las zonas de estudio, lo cual se observa en algunos centros de playa tales como La Paz, Loreto y Los Cabos, Baja California Sur. En contraste, un coeficiente menor que la unidad sugiere un menor crecimiento respecto al promedio, como el que se presenta en Acapulco, Guerrero, San Carlos-Empalme, Sonora, y Mazatlán, Sinaloa.

Cuadro 2

Crecimiento Estimado del PIB en las Principales Zonas Turísticas de Playa, 1993 - 2017

\begin{tabular}{|c|c|c|c|c|}
\hline \multirow{2}{*}{ Zona Turística } & \multicolumn{3}{|c|}{ Luminosidad por km2 } & \multirow{2}{*}{$\begin{array}{c}\text { Crecimiento } \\
\text { Relativo }^{*} \\
\text { 1993-2017 } \\
\end{array}$} \\
\hline & 1993 & 2013 & 2017 & \\
\hline Los Cabos & 1.42 & 5.12 & 5.84 & 1.15 \\
\hline Loreto & 0.18 & 0.40 & 0.99 & 1.14 \\
\hline La Paz & 0.48 & 1.12 & 1.92 & 1.14 \\
\hline Chetumal & 0.59 & 1. 16 & 1.97 & 1.12 \\
\hline Puerto Escondido & 1.93 & 4.52 & 5.52 & 1.11 \\
\hline Cancún-Riviera Maya & 2.18 & 5.63 & 6.70 & 1. 10 \\
\hline Manzanillo-Costa Alegre & 4.89 & 8.99 & 11.50 & 1.07 \\
\hline Vallarta- Riviera Nayarit & 5.65 & 12.29 & 13.36 & 1.03 \\
\hline Playas de Rosarito & 7.28 & 15.55 & 19.13 & 1.02 \\
\hline Veracruz-Boca del Rio & 19.89 & 34.13 & 35.21 & 0.92 \\
\hline Ixtapa Zihuatanejo & 2.88 & 4.14 & 3.99 & 0.90 \\
\hline Bahias de Huatulco & 11.22 & 13.58 & 16.89 & 0.89 \\
\hline Mazatlán & 18.80 & 29.23 & 31.64 & 0.88 \\
\hline San Carlos- Empalme & 14.28 & 22.55 & 21.54 & 0.87 \\
\hline Acapulco & 21.54 & 26.91 & 22.21 & 0.75 \\
\hline
\end{tabular}

Nota: La selección se realizó con base en la lista de centros de playa que publica la Sectur en el Monitoreo Hotelero de 70 Centros Turísticos.

Fuente: Estimaciones propias con base en información del INEGI, el Departamento de Defensa de Estados Unidos y el Earth Observation Group, NOAA National Geophysical Data Center.

\footnotetext{
${ }^{7}$ Para el periodo posterior a 2013 se utilizó la elasticidad obtenida para el periodo 1993 - 2013, pero con los datos de luminosidad correspondientes al año de estimación.
} 


\section{Discusión de Los Resultados}

Nuestras estimaciones indican que existe una gran heterogeneidad en el grado de crecimiento económico entre los distintos destinos de playa en México y que pueden estar influenciado por múltiples factores, entre los que destacan dos por su importancia: Las zonas turísticas de playa orientadas al turismo receptivo y los niveles de inseguridad en dichas zonas.

En efecto, es de hacerse notar que aquellas zonas turísticas de playa que registraron los mayores crecimientos tienden a estar más orientadas al turismo receptivo: la relación entre el crecimiento económico y la proporción de pasajeros internacionales que llegan a cada destino es positiva, como se puede apreciar en la Gráfica 2.

\section{Gráfica 2}

Relación entre el Crecimiento de la Actividad y la Participación de los Pasajeros Internacionales por Zona Turística

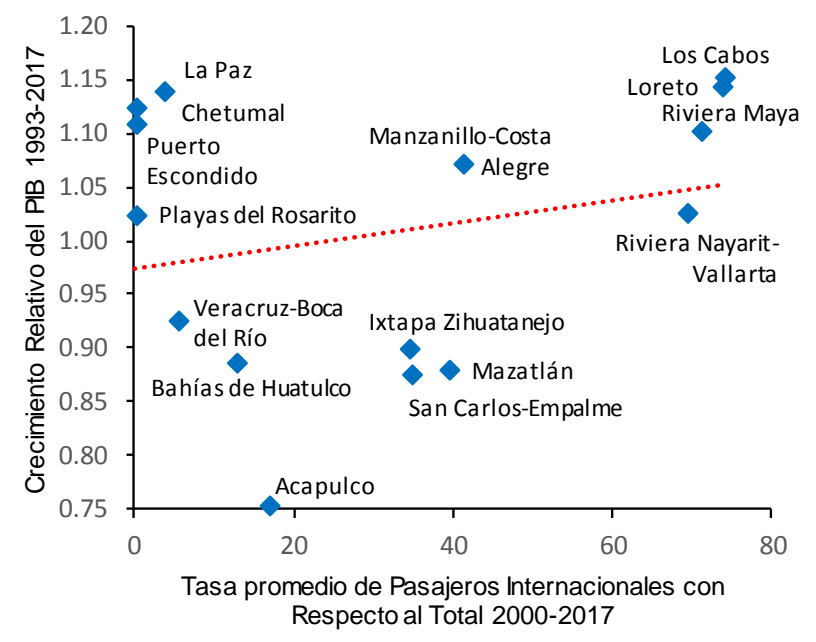

Fuente: Estimaciones propias con datos de Aeropuertos y Servicios Auxiliares.

La evidencia grafica indica que para los casos de los aeropuertos de Chetumal, La Paz y Playas del Rosarito, la llegada de pasajeros internacionales es baja, lo que podría deberse a su cercanía con aeropuertos internacionales de gran envergadura como Playas del Rosarito/Tijuana - San Diego; Chetumal/Cancún y La Paz/Los Cabos. No obstante, aún con estas observaciones, se puede distinguir una relación positiva entre la tasa promedio de pasajeros internacionales con respecto al total y el crecimiento relativo del PIB.

Si bien estudios previos sugieren que el turismo receptivo depende en gran medida de factores externos como el PIB de Estados Unidos y Canadá (Guzmán et al 2011) o el tipo de 
Cambio (Sánchez y Cruz 2016), también depende de otros factores, como lo mencionan los contactos empresariales del sector turismo entrevistados para el Reporte de Economías Regionales del Banco de México (2018), quienes mencionan que los centros turísticos que han sido exitosos en atraer al turismo internacional se caracterizan por su mayor conectividad aérea y por implementar estrategias de publicidad y promoción en el extranjero a través de organismos locales de fideicomisos que administran los impuestos al hospedaje. Asimismo, estos destinos han incrementado su infraestructura turística, en particular aquella relacionada con la pesca deportiva, los campos de golf y otras amenidades. Adicionalmente, los contactos corroboran que la depreciación del tipo de cambio también ha derivado en una mayor competitividad de las zonas turísticas en México, en relación con otros destinos internacionales.

Estas diferencias en el desempeño económico de las zonas turísticas de playa también pueden estar relacionadas con otros factores; en particular, con los niveles de inseguridad (De la Torre y Navarrete, 2013). Midiendo inseguridad a través de la tasa de homicidios por cada cien mil habitantes ${ }^{8}$ el crecimiento en la actividad de las zonas turísticas está asociado de manera negativa con la inseguridad (Gráfica 3). Cabe destacar el caso de Acapulco, el cual está muy por encima en la medición de inseguridad y es el destino de playa estudiado con menor crecimiento en su actividad económica. Esta información también es corroborada por los directivos empresariales entrevistados por el citado Reporte de Economías Regionales del Banco de México (2018), quienes mencionan que la demanda por sus servicios se ve afectada negativamente por los niveles de violencia e inseguridad en la localidad.

\footnotetext{
${ }^{8}$ La tasa de homicidios intencionales es una medición de inseguridad más certera, debido a su baja incidencia de cifra negra, es decir, el número de delitos que no es denunciado o reportado a las autoridades.
} 


\section{Gráfica 3}

Relación entre el Crecimiento de la Actividad y los Homicidios por cada 100,000 habitantes por Zona

Turística.

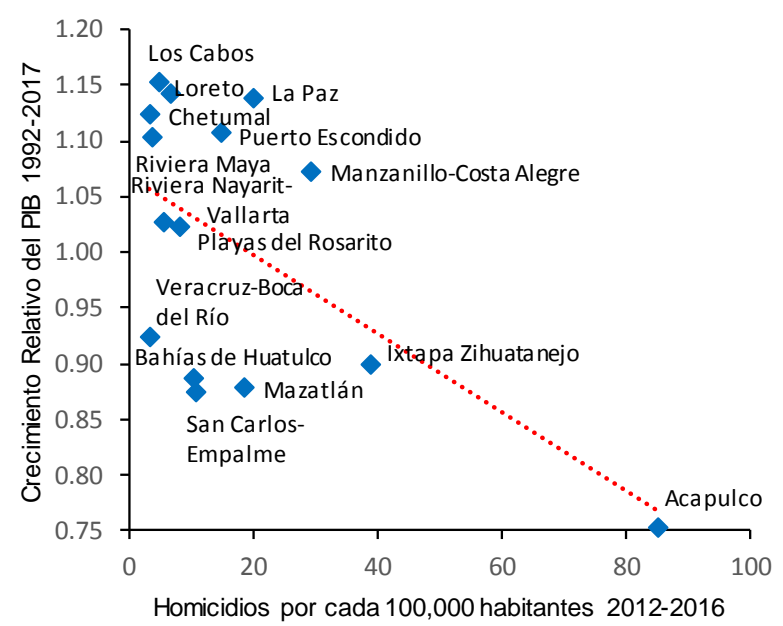

Fuente: Estimaciones propias con datos de la Estadística de Defunciones Generales del INEGI.

\section{Conclusiones}

Los resultados del presente documento de investigación indican que existe una gran heterogeneidad en el crecimiento económico de las principales zonas de playa en México. Las áreas de mayor crecimiento se encuentran localizadas principalmente en la región centro norte del país, específicamente en Baja California Sur, si bien Chetumal y Cancún-Riviera Maya en Quintana Roo y Puerto Escondido en Oaxaca también registraron crecimientos relativamente elevados. En contraste, las zonas turísticas de playa que registraron el menor dinamismo económico son Acapulco e Ixtapa Zihuatanejo, Guerrero; Bahías de Huatulco, Oaxaca; San Carlos-Empalme, Sonora; y Mazatlán, Sinaloa.

Se observa que las zonas que registraron un mayor dinamismo durante el periodo analizado son aquellas que tienden a estar más orientadas en atraer a viajeros internacionales. En contraste, se detectó una correlación negativa entre el desempeño económico de las zonas turísticas y la inseguridad (medida por la tasa de homicidios por cada cien mil habitantes). Así, resulta fundamental seguir implementado acciones que impulsen la atracción del turismo internacional a través de una mejor conectividad aérea y terrestre, el desarrollo de infraestructura turística, la promoción de los destinos turísticos y una mejora en las condiciones de seguridad. Esto redundaría en un mayor crecimiento de algunas zonas 
turísticas, ubicadas principalmente en el sur, que no han mostrado el mismo dinamismo que otras.

Finalmente, cabe destacar que la utilización de imágenes satelitales abre nuevas posibilidades para el análisis regional, ya que permite medir la evolución de la actividad económica en un nivel menos agregado, y en algunos casos con mayor oportunidad, que la información oficial disponible, lo cual facilita, en particular, el estudio del impacto de fenómenos coyunturales y de políticas públicas sobre el desempeño económico regional. 


\section{Bibliografía.}

Banco de México (2018). Reporte sobre las Economías Regionales, Enero-Marzo 2018.

Cárdenas Gómez, Erika Patricia. (2016) "Crecimiento y Planeación Urbana en Acapulco, Cancún y Puerto Vallarta (México)”. Revista Investigaciones Turísticas, 12:99-120.

Chen, Xi; Nordhaus, William D. (2011) "Using luminosity data as a proxy for economic statistics". Proceedings of the National Academy of Sciences May 2011, 108 (21) 8589-8594.

De la Torre M. y D. Navarrete (2013). "Turismo Violencia y Vulnerabilidad." Kairos, Revista de Temas Sociales, 17(31) Mayo:1-13.

Doll, Christopher; Muller, Jan-Peter; Morley, Jeremy G. (2006) "Mapping Regional Economic Activity from Night-Time Light Satellite Imagery". Ecological Economics 57: 75-92.

Guzmán Soria, Eugenio; Rebollar Rebollar, Samuel; Hernández Martínez, Juvencio; De la Garza Carranza, María Teresa; García Salazar, José Alberto. (2011) "Factores Determinantes de la Demanda Internacional del Turismo en México". GCG: Revista de Globalización, Competitividad y Gobernabilidad. 5(3): 30-49.

Harari, M. (2015). "Cities in bad shape: urban geometry in India", Manuscrito, Massachusetts Institute of Technology

Henderson, J. Vernon; Storeygard, Adam; Weil, David N. (2012) "Measuring Economic Growth from Outer Space". American Economic Review 102(2):994-1028.

Montaño Armendáriz, Angélica; Pérez Concha, Juan Carlos; Burrola; Verónica de la O. "Crisis Económica Internacional y Turismo en México: Impacto y Alternativas para los Destinos Turísticos de Playa”. Manuscrito.

Rassy, Dunia; Smith, Richard D. (2013) “The Economic Impact of H1N1 on México's Tourist and Pork Sectors". Health Economics, 22:824-834.

Sánchez López, Fenando; Cruz Marcelo, José Nabor. (2016) “Determinantes Económicos de los Flujos de Viajeros a México". Revista de Análisis Económico, 33(2):3-36. 
Sutton, Paul; Elvidge, Christopher; Tilottama, Ghosh. (2007). "Estimation of Gross Domestic Product at Sub-National Scales Using Nighttime Satellite Imagery". International Journal of Ecological Economics \& Statistics. 8. 